Modeling hydroxylated nanosilica: Testing the performance of ReaxFF and FFSiOH force fields

Antoni Macià Escatllar, Piero Ugliengo, and Stefan T. Bromley

Citation: The Journal of Chemical Physics 146, 224704 (2017); doi: 10.1063/1.4985083

View online: http://dx.doi.org/10.1063/1.4985083

View Table of Contents: http://aip.scitation.org/toc/jcp/146/22

Published by the American Institute of Physics

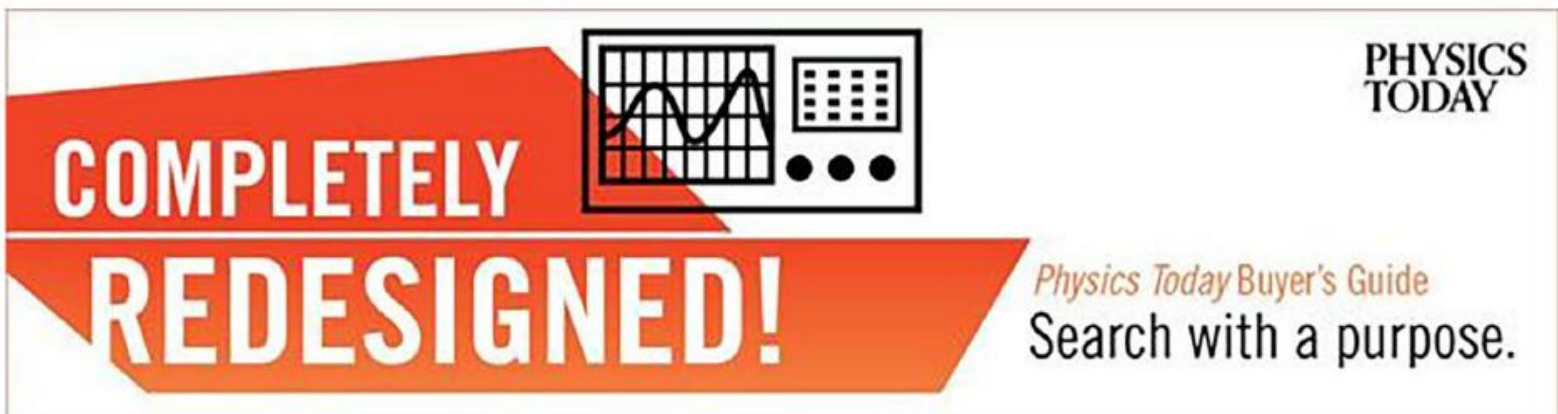




\title{
Modeling hydroxylated nanosilica: Testing the performance of ReaxFF and FFSiOH force fields
}

\author{
Antoni Macià Escatllar, ${ }^{1}$ Piero Ugliengo, ${ }^{2}$ and Stefan T. Bromley ${ }^{1,3, a)}$ \\ ${ }^{1}$ Departament de Ciència de Materials i Química Física and Institut de Química Teòrica $i$ \\ Computacional (IQTCUB), Universitat de Barcelona, E-08028 Barcelona, Spain \\ ${ }^{2}$ Dipartimento di Chimica and NIS Centre, Università degli Studi di Torino, 10125 Torino, Italy \\ ${ }^{3}$ Institució Catalana de Recerca i Estudis Avançats (ICREA), E-08010 Barcelona, Spain
}

(Received 30 March 2017; accepted 24 May 2017; published online 13 June 2017)

\begin{abstract}
We analyze the performance of the FFSiOH force field and two parameterisations of the ReaxFF force field for modeling hydroxylated nanoscale silica $\left(\mathrm{SiO}_{2}\right)$. Such nanosystems are fundamental in numerous aspects of geochemistry and astrochemistry and also play a key role during the hydrothermal synthesis of technologically important nanoporous silicas (e.g., catalysts, absorbents, and coatings). We consider four aspects: structure, relative energies, vibrational spectra, and hydroxylation energies, and compare the results with those from density functional calculations employing a newly defined dataset (HND: Hydroxylated Nanosilica Dataset). The HND consists of three sets of $\left(\mathrm{SiO}_{2}\right)_{16}\left(\mathrm{H}_{2} \mathrm{O}\right)_{\mathrm{N}}$ nanoparticles (NPs), each with a different degree of hydroxylation and each containing between 23 and 26 distinct isomers and conformers. We also make all HND reference data openly available. We further consider hydroxylated silica NPs of composition $\left(\mathrm{SiO}_{2}\right)_{\mathrm{M}}\left(\mathrm{H}_{2} \mathrm{O}\right)_{\mathrm{N}}$ with $\mathrm{M}=4,8,16$, and 24 and infinite surface slabs of amorphous silica, both with variable hydroxylation. For energetics, both ReaxFF and FFSiOH perform well for NPs with an intermediate degree of hydroxylation. For increased hydroxylation, the performance of FFSiOH begins to significantly decline. Conversely, for the lower degree of hydroxylation both parameterisations of ReaxFF do not perform well. For vibrational frequencies, FFSiOH performs particularly well and significantly better than ReaxFF. This feature also opens the door to inexpensively calculating Gibbs free energies of the hydroxylated nanosilica systems in order to efficiently correct density functional theory calculated electronic energies. We also show how some small changes to FFSiOH could improve its performance for higher degrees of hydroxylation. Published by AIP Publishing. [http://dx.doi.org/10.1063/1.4985083]
\end{abstract}

\section{INTRODUCTION}

The intimate relation between water and nanoscale silica $\left(\mathrm{SiO}_{2}\right)$ together with the power and versatility of sol-gel synthesis techniques has led to many technological applications and materials (e.g., optical coatings, drug delivery, and mesoporous silica) based on hydroxylated silica nanoparticles (NPs) and their coalescence. ${ }^{1,2}$ Nature also takes advantage of this general approach with exquisite enzymatic control of biomineralization to form many types of condensed biosilicas. ${ }^{3}$ Hydrated silica NPs are also widely used as fillers to enhance the physical and mechanical properties of other materials (e.g., cement-based materials, ${ }^{4}$ coatings, ${ }^{5}$ and polymers $^{6}$ ). Understanding interactions between silica and water at the nanometre and sub-nanometre scale has also led to fundamental insights into the formation of mesoporous zeolites, ${ }^{7}$ geological weathering of silicate minerals, ${ }^{8}$ and the aqueous corrosion of silica-based materials. ${ }^{9}$ The nanosilicawater interface is also important to understand the formation of ice-covered silicate dust grains in an astrochemical context. ${ }^{10}$

The degree of hydroxylation (i.e., the density of hydroxyl groups) plays a very important role with respect to both

\footnotetext{
a) Author to whom correspondence should be addressed: s.bromley@ub.edu
}

the structure and properties of nanosilica. Although the link between hydroxyl coverage and surface adsorption has long been established, ${ }^{11}$ there is still a need to understand silicawater interactions and their technological and biological implications at the molecular scale and nanoscale. It has been shown, for example, that the proportion of hydroxyls on small silica particles can be deliberately increased by physico-chemical processing to produce enhanced properties for applications (e.g., mechanically robust antireflective films ${ }^{12}$ and epoxidation catalysis ${ }^{13}$ ). For particularly well-defined silica nanoslabs, even the spatial distribution of hydroxyls can be tuned experimentally with potential for templating supported species. ${ }^{14}$ The surface hydroxyl density has also been reported to influence the toxicity and biocompatibility of silica NPs. ${ }^{15-17}$ Experimentally, the water-nanosilica interface has been the focus of numerous experimental and theoretical studies, ${ }^{18-20}$ with empirical models describing the surface hydroxylation chemistry of silica having been put forward. ${ }^{21,22}$ However, such models fall short of providing a truly microscopically detailed general explanatory account of this important system.

Atomistically detailed insights into the growth, chemistry, and structure of molecular and nanoscale hydroxylated silica have been provided by bottom-up computational modelling approaches. Ab initio quantum chemical methods have been 
used to extract information on the thermodynamics of $\mathrm{Si}(\mathrm{OH})_{4}$ monomers and their oligomerization. ${ }^{23-26}$ For considerably larger hydroxylated nanosilica systems, typically involving thousands of atoms, classical force field methods have also been applied. ${ }^{12,27,28}$ Although numerous force fields have been proposed in the literature for modeling such large nanosilicawater systems and/or bulk silica surfaces interacting with water, ${ }^{19,29-37}$ very few force fields have been tested to some degree with respect to their adequacy for treating hydroxylated nanosilica systems possessing of the order of 100 atoms. ${ }^{19,38}$ The size regime for systems possessing between $10 \mathrm{~s}$ and $100 \mathrm{~s}$ of atoms is particularly important as it addresses the transition between molecular and bulk scales. ${ }^{39}$ This size range is especially relevant for understanding growth, dissolution, and the bottom-up emergence of the nanoscale structure and properties with increasing size. Hydroxylated nanosilica systems with sizes toward the lower end of this size range have been shown to be well within the reach of modern parallel implementations of $a b$ initio methods such as density functional theory (DFT). ${ }^{40-43}$ However, for larger nanosystems and for problems requiring $>1$ ns time scales to be monitored (e.g., molecular dynamics) or where extensive rapid sampling of 1000s of structures is required (e.g., Monte Carlo and global optimisation), a suitably accurate force field is required.

Herein we test the performance of the ReaxFF force field, ${ }^{29}$ its recent re-parameterisation, ${ }^{27}$ and the $\mathrm{FFSiOH}^{36}$ force field for describing the properties of intermediate sized hydroxylated silica NPs. The ReaxFF force fields contain a relatively large number of parameters and aim to describe both the properties of water and silica and the reactions between them with an accuracy approaching that of DFT calculations. The simpler FFSiOH force field does not describe aqueous phases but has shown to reproduce the structure and relative phase stability of bulk phases of both hydroxylated and anhydrous silica and to provide good models of amorphous silica surfaces. FFSiOH also has been shown to be capable of providing accurate infrared (IR) vibrational frequencies. We compare the energetic and structural predictions of ReaxFF and FFSiOH with suitable DFT calculated data and provide an assessment of their strengths and weaknesses for modeling hydroxylated silica NPs.

\section{METHODOLOGY}

In order to assess the performance of ReaxFF and FFSiOH to describe the properties of hydroxylated silica NPs, we require a benchmark set of reference data to compare with. Previous work has shown that DFT calculations using the B3LYP $^{44}$ functional and a $6-31 G(d, p)$ basis set provide a good compromise between computational efficiency and accuracy for hydroxylated silica systems. ${ }^{25,41,45}$ For our analysis, we employ B3LYP/6-31G(d,p)-calculated structures, relative energetics, and IR vibrational frequencies of hydroxylated silica NPs containing up to 108 atoms. For all DFT calculations, the Gaussian 09 code $^{46}$ was employed.

Specifically, for examining hydroxylation energies we employ NPs of sizes $\left(\mathrm{SiO}_{2}\right)_{4}\left(\mathrm{H}_{2} \mathrm{O}\right)_{\mathrm{M}},\left(\mathrm{SiO}_{2}\right)_{8}\left(\mathrm{H}_{2} \mathrm{O}\right)_{\mathrm{M}}$, $\left(\mathrm{SiO}_{2}\right)_{16}\left(\mathrm{H}_{2} \mathrm{O}\right)_{\mathrm{M}}$, and $\left(\mathrm{SiO}_{2}\right)_{24}\left(\mathrm{H}_{2} \mathrm{O}\right)_{\mathrm{M}}$ with varying $\mathrm{M}^{41,42}$ and five extended silica nanoslabs with varying degrees of hydroxylation. ${ }^{45}$ For a more detailed analysis of structure and relative energies, we focus mainly on an extensive dataset of over $60\left(\mathrm{SiO}_{2}\right)_{16}\left(\mathrm{H}_{2} \mathrm{O}\right)_{\mathrm{N}}$ isomers containing between 60 and 72 atoms. We note that a small proportion of these are strictly conformers (i.e., where the $\mathrm{Si}-\mathrm{O}$ framework topology is maintained but where the $\mathrm{OH} \cdots \mathrm{OH} \mathrm{H}$-bonding network differs). All these NPs were derived using global optimisation as detailed in the previous works, ${ }^{40-43,50}$ specifically for this study. The nanocluster structures taken from our global optimisations are those found to have the lowest energy as calculated using DFT and are thus relatively unbiased with respect to the force field parameterisation. Obtaining hydroxylated structures via modeling the reaction of silica nanoclusters with water molecules (via ReaxFF or DFT) could also provide a dataset for comparative studies but would: (i) not guarantee finding particularly low energy nanoclusters, and (ii) (in the case of using ReaxFF) tend to bias the dataset to those systems most energetically favored by the respective ReaxFF parameterisation. We collate all cluster structures and energies in the supplementary material which we make openly available as a resource (the HND - "Hydroxylated Nanosilica Dataset") for testing and parameterising other modeling methods (e.g., new force fields). We note that the full parameterisation of FFSiOH and much of that of ReaxFF was based on comparisons with data from DFT calculations using the B3LYP functional and thus our full reference set of structures and relative energies (all obtained from B3LYP/6-31G(d,p) calculations) is particularly suitable for the present analysis.

Due to the relatively large set of $\left(\mathrm{SiO}_{2}\right)_{16}\left(\mathrm{H}_{2} \mathrm{O}\right)_{\mathrm{N}}$ species, containing, for each $\mathrm{N}$ subset, a large number of distinct local structural features, we use these NPs for obtaining statistical distributions of various chemically meaningful geometrical parameters. We note that in the case of $\mathrm{Si}-\mathrm{O}$ bond lengths, $\mathrm{O}-$ $\mathrm{Si}-\mathrm{O}$, and $\mathrm{O}-\mathrm{Si}-\mathrm{O}$ angles, each subset possesses over 1500 distinct data points, whereas for the parameters involving surface $\mathrm{OH}$ groups, the number is reduced to between 95 and 400 , depending on the degree of hydroxylation. The HND, consisting of $\left(\mathrm{SiO}_{2}\right)_{16}\left(\mathrm{H}_{2} \mathrm{O}\right)_{\mathrm{N}}$ isomers, is divided into three similarly sized subsets, each having the same stoichiometry (i.e., for $\mathrm{N}=4,6,8$ ). For NPs, we define the degree of hydroxylation by the percentage of dissociatively chemisorbed water molecules with respect to the total number of $\mathrm{SiO}_{2}$ units (i.e., $\mathrm{R}=\mathrm{N} / \mathrm{M}$ ). A selection of NPs from the HND are shown in Fig. 1. Informally, these three degrees of hydroxylation can also be considered to be varying between low, intermediate, and high degrees of hydroxylation. The $\mathrm{N}=4$ set can be regarded as having a relatively low $25 \%$ degree of hydroxylation. All NPs in this low hydroxylation sub-set, as for the full set of all $\left(\mathrm{SiO}_{2}\right)_{16}\left(\mathrm{H}_{2} \mathrm{O}\right)_{\mathrm{N}}$ isomers considered, do not exhibit terminal oxygen defects. However, at this low degree of hydroxylation, many NPs possess rather strained threemembered $(\mathrm{SiO})_{3}$ rings ${ }^{47}$ indicating that further ring-opening via hydroxylation would be energetically favourable. ${ }^{41}$ The $\mathrm{N}=8$ sub-set of NPs can be regarded as having a high $50 \%$ degree of hydroxylation and often exhibit between one and three geminal silanol groups (i.e., two hydroxyls bound to one silicon atom). Considering the low percentage of geminal sites estimated to typically exist on bulk amorphous silica surfaces, ${ }^{17}$ this degree of NP hydroxylation can indeed be 


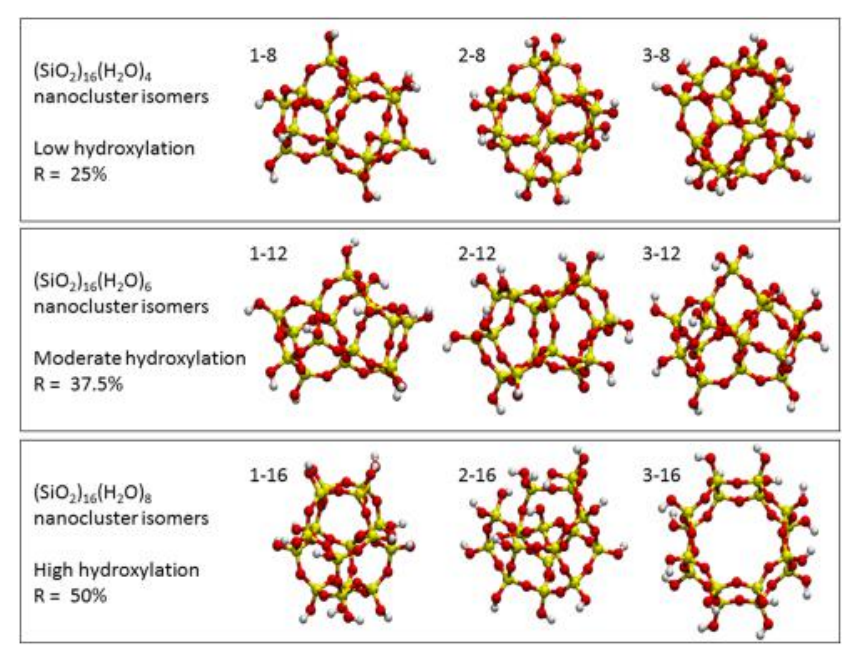

FIG. 1. Example isomer structures from the HND - Hydroxylated Silica Dataset (see the supplementary material). The lowest energy isomer for each degree of hydroxylation is shown to the left of each set of three. The two other isomers shown for each set are example higher energy isomers. The degree of hydroxylation of each set of $\left(\mathrm{SiO}_{2}\right)_{16}\left(\mathrm{H}_{2} \mathrm{O}\right)_{\mathrm{N}} \mathrm{NPs}$ is defined to be $\mathrm{R}=\mathrm{N} / 16$. Element colour key: silicon—yellow, oxygen—red, and hydrogen—grey.

considered very high. We note that for such a high level of hydroxylation, one can also find low energy $\left(\mathrm{SiO}_{2}\right)_{16}\left(\mathrm{H}_{2} \mathrm{O}\right)_{8}$ isomers with a hydrogen bonded water molecule instead of two extra hydroxyls. ${ }^{41}$ In this study we exclude such isomers as in the FFSiOH force field, the free water molecule and its interactions are not explicitly parameterised. However, we note that such cases could make an interesting extended test for the ReaxFF force fields. Estimates of the optimal degree of hydroxylation for $\left(\mathrm{SiO}_{2}\right)_{16}\left(\mathrm{H}_{2} \mathrm{O}\right)_{\mathrm{N}}$ isomers give $\mathrm{R} \sim 0.315,{ }^{41}$ close to our class of moderately hydroxylated NPs with R $=0.375$. This $\mathrm{N}=6$ set of NPs has an intermediate degree of hydroxylation and does not display three-membered rings or geminal silanols, indicating that these NPs possess a rather well balanced hydroxylated structure. From this full set, we derive distributions of various local structural features (e.g., $\mathrm{Si}-\mathrm{O}$ distances, $\mathrm{Si}-\mathrm{O}-\mathrm{Si}$ angles, $\mathrm{O}-\mathrm{Si}-\mathrm{O}$ angles) and relative energies of stoichiometrically similar isomers from the three considered force fields and compare this data with the corresponding DFT-calculated data. In addition, we use the lowest energy isomer of each of the different hydroxylated subsets to calculate IR frequencies. All force field calculations were performed using the GULP code. ${ }^{48}$

\section{RESULTS AND DISCUSSION}

\section{A. Hydroxylation energies}

We first report on the normalised hydroxylation energies $\left(\mathrm{E}_{\text {hydrox }}\right)$ of $\left(\mathrm{SiO}_{2}\right)_{\mathrm{M}}\left(\mathrm{H}_{2} \mathrm{O}\right)_{\mathrm{N}}\left(\mathrm{M}=4,8,16\right.$, and 24) $\mathrm{NPs}^{41}$ and a $1.5 \mathrm{~nm}$ thick nanoslab. For a system of size $\mathrm{M}$ units, we have

$$
E_{\text {hydrox }}=\frac{E\left[\left(\mathrm{SiO}_{2}\right)_{\mathrm{M}}\left(\mathrm{H}_{2} \mathrm{O}\right)_{\mathrm{N}}\right]+(M-N) \cdot E\left[\mathrm{H}_{2} \mathrm{O}\right]}{M}-E_{\text {ref }} \text {, }
$$

where $\mathrm{E}_{\mathrm{ref}}$ for the NPs is taken to be the energy of the normalised bare four unit silica cluster and four water molecules

$$
E_{\text {ref }}=\frac{E\left[\left(\mathrm{SiO}_{2}\right)_{4}\right]+4 \cdot E\left[\mathrm{H}_{2} \mathrm{O}\right]}{4}
$$

calculated by the respective method (i.e., force field type or DFT). Although, unlike ReaxFF, FFSiOH is not formally parameterised for the treatment of water molecules and their interactions, it still provides a sensible energy for an isolated $\mathrm{H}_{2} \mathrm{O}$ species. Thus, for consistency, for each set of calculations, we take $E\left(\mathrm{H}_{2} \mathrm{O}\right)$ to be that of the optimised $\mathrm{H}_{2} \mathrm{O}$ species as calculated by the corresponding method. For brevity and clarity, we distinguish the two versions of ReaxFF by use of the following nomenclature: ReaxFF1 (original parameterisation by Fogarty et al. $^{29}$ ) and ReaxFF2 (re-parameterisation by Yeon and van Duin ${ }^{27}$ ).

The general picture for the NPs [Fig. 2(a)] shows that the $\mathrm{E}_{\text {hydrox }}$ values from all force fields follow the DFT trend of decreasing with both cluster size and degree of hydroxylation. The majority of $\mathrm{E}_{\text {hydrox }}$ values given by $\mathrm{FFSiOH}$ are more negative than the DFT values by $\sim 10-20 \mathrm{~kJ} / \mathrm{mol}$ for low to moderate degrees of hydroxylation. This difference tends to become larger for higher degrees of hydroxylation, especially for the smaller NPs where it reaches a maximum of $53 \mathrm{~kJ} / \mathrm{mol}$ for the most hydroxylated ( $M=4) \mathrm{NP}$. This effect is likely to be at least partially due to the FFSiOH value of $E\left(\mathrm{H}_{2} \mathrm{O}\right)$ which, not being a parameterised value for the water molecule, leads to a small but cumulative divergence away from the DFT data with increasing hydroxylation. We note, however, that this effect is much less evident for the larger sized nanoclusters (i.e., for $\mathrm{N}$ $=16,24)$.

Both ReaxFF force fields behave similarly in terms of their overall pattern of $E_{\text {hydrox }}$ variation with respect to increasing hydroxylation for each NP size. Little cumulative divergence away from the DFT calculated data is evident consistent with the parameterised capacity of ReaxFF to deal with water molecules. ReaxFF1, however, generally predicts significantly more negative $\mathrm{E}_{\text {hydrox }}$ values than ReaxFF2 (by $\sim 80 \mathrm{~kJ} / \mathrm{mol}$ ). This difference between the two versions of ReaxFF is all the more significant as the re-parameterised ReaxFF2 force field generally predicts remarkably good $\mathrm{E}_{\text {hydrox }}$ values with respect to the DFT-calculated data, meaning that ReaxFF1 seriously overestimates $\mathrm{E}_{\text {hydrox }}$ magnitudes. The predicted $\mathrm{E}_{\text {hydrox }}$ values from ReaxFF2 are particularly good for small cluster sizes of $\mathrm{M}=4$ and 8 (typically within $\sim 8 \mathrm{~kJ} / \mathrm{mol}$ of the DFT-calculated values). For the larger sized NPs, the performance of ReaxFF2 becomes similar to, albeit arguably slightly better than, that of FFSiOH.

In the case of the hydroxylated amorphous silica nanoslab [Fig. 2(b)], all force fields seem to perform similarly well, with the overestimation of $E_{\text {hydrox }}$ magnitudes found for the NPs also observed. Here, the energy difference between the $\mathrm{E}_{\text {hydrox }}$ values predicted by DFT and the force fields (where $E_{\text {ref }}$ is set to be the respective energy of the most poorly hydroxylated nanoslab) increases with increasing hydroxylation and reaches a maximum of $23 \mathrm{~kJ} / \mathrm{mol}$ at the highest degree of hydroxylation. Note that for this system, we follow the experimental convention and define the degree of hydroxylation in terms of the number of hydroxyls per square nanometre. Interestingly, for this system, all force fields perform almost identically up until the highest considered degree of hydroxylation, where the overestimation of $E_{\text {hydrox }}$ magnitudes is slightly higher for FFSiOH than for ReaxFF1 and ReaxFF2. 


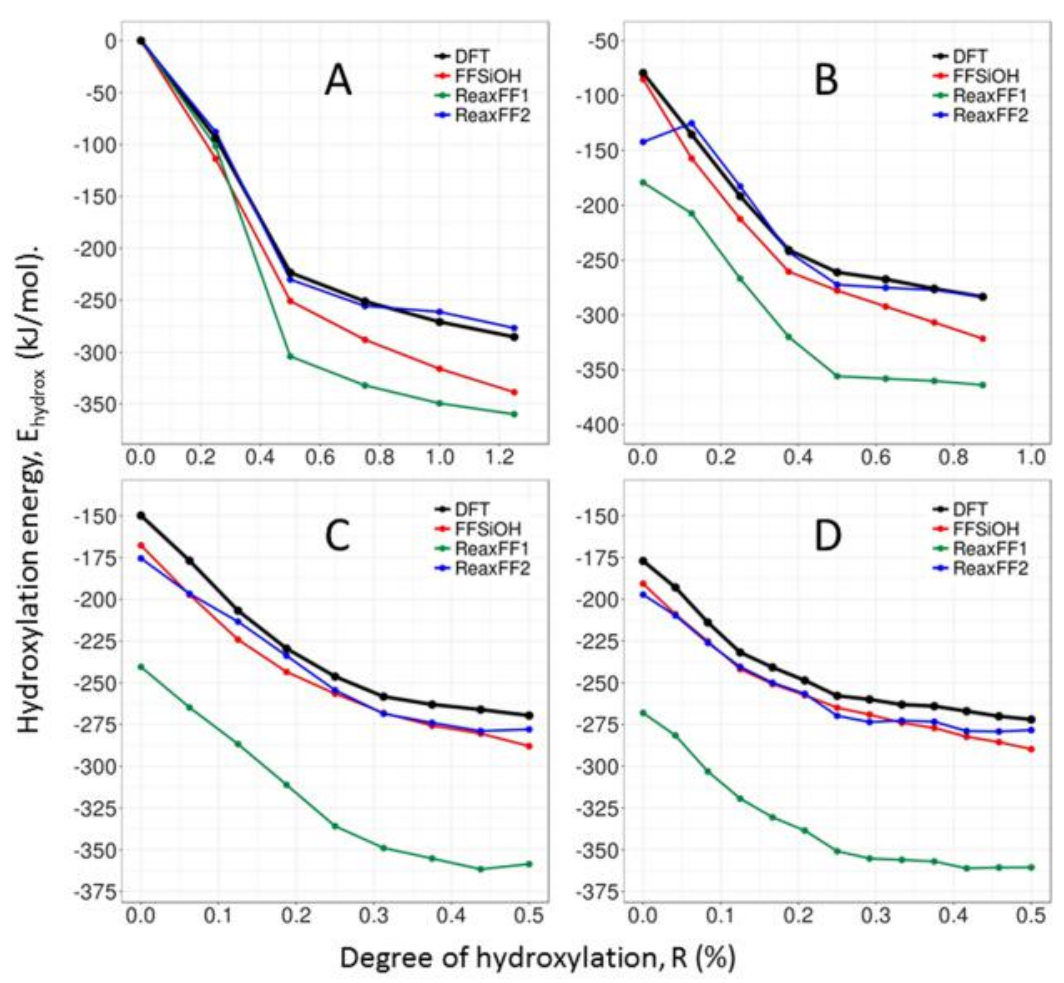

(a)

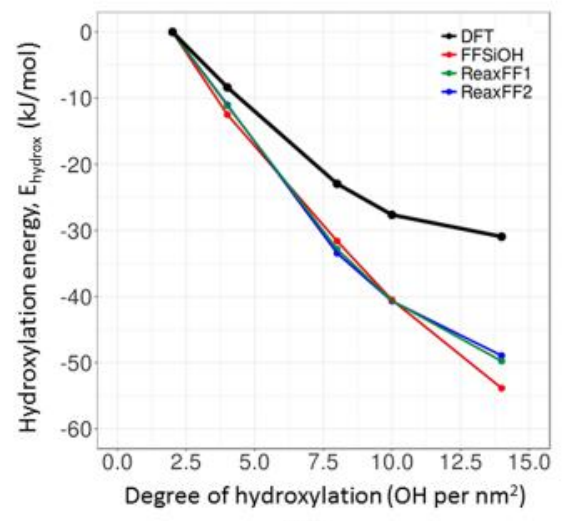

(b)
FIG. 2. (a) Plots of hydroxylation energy ( $E_{\text {hydrox }}$ ) as calculated by DFT (black), FFSiOH (red), ReaxFF1 (green), and ReaxFF2 (blue) for (A) $\left(\mathrm{SiO}_{2}\right)_{4}\left(\mathrm{H}_{2} \mathrm{O}\right)_{\mathrm{N}} \mathrm{NPs}$, (B) $\left(\mathrm{SiO}_{2}\right)_{8}\left(\mathrm{H}_{2} \mathrm{O}\right)_{\mathrm{N}} \mathrm{NPs}$, (C) $\left(\mathrm{SiO}_{2}\right)_{16}\left(\mathrm{H}_{2} \mathrm{O}\right)_{\mathrm{N}} \mathrm{NPs}$, and (D) $\left(\mathrm{SiO}_{2}\right)_{24}\left(\mathrm{H}_{2} \mathrm{O}\right)_{\mathrm{N}} \mathrm{NPs}$. The degree of hydroxylation is defined by $\mathrm{R}(=\mathrm{N} / \mathrm{M})$. (b) Plot of hydroxylation energy $\left(\mathrm{E}_{\text {hydrox }}\right)$ with respect to the degree of hydroxylation as calculated by DFT (black), FFSiOH (red), ReaxFF1 (green), and ReaxFF2 (blue) for a $1.5 \mathrm{~nm}$ thick amorphous silica nanoslab.

\section{B. Distributions of local structure features}

\section{O-Si-O angles}

In silica, the $\mathrm{O}-\mathrm{Si}-\mathrm{O}$ angle is largely constrained by the rigid tetrahedrality of the $\mathrm{SiO}_{4}$ sub-units. In pure silica, these tetrahedra can be distorted by highly strained small $(\mathrm{SiO})_{\mathrm{n}}$ rings ${ }^{47,49}$ or terminating defects ${ }^{50,51}$ typically found in anhydrous silica. As low barrier reactions with water are generally favoured at such sites, quickly leading to ring opening or healing of terminating defects, ${ }^{52-56}$ it is expected that the $\mathrm{O}-\mathrm{Si}-\mathrm{O}$ angle distribution in nanosilica should be relatively narrow and invariant above a certain degree of hydroxylation. In Fig. 3, we show the distribution of $\mathrm{O}-\mathrm{Si}-\mathrm{O}$ angles for our hydroxylated nanosilica dataset, which consists of $\left(\mathrm{SiO}_{2}\right)_{16}\left(\mathrm{H}_{2} \mathrm{O}\right)_{\mathrm{N}}$ isomers with low $(\mathrm{N}=4, \mathrm{R}=0.25 \%)$, intermediate $(\mathrm{N}=6$, $\mathrm{R}=0.375 \%)$, and high $(\mathrm{N}=8, \mathrm{R}=0.5 \%)$ levels of hydroxylation, for clusters optimised using the three considered force fields and DFT. In all cases, a narrow distribution of $\mathrm{O}-\mathrm{Si}-\mathrm{O}$ angles centred close to the ideal tetrahedral angle of $109.5^{\circ}$ is found. Upon close inspection of the height, breadth, and form of the force field derived distributions, it can be seen that the FFSiOH optimised clusters have sharp single peaked O-Si-O distributions which are very similar to those derived from the DFT-optimised NP structures. Conversely, the distributions from the NPs optimised using both versions of ReaxFF are lower and wider than the DFT-derived distributions. For the NPs with a low degree of hydroxylation, this is mainly apparent for angles smaller than $109.5^{\circ}$. Here, although both DFT and FFSiOH distributions quickly drop to zero going from $105^{\circ}$ to $100^{\circ}$, both ReaxFF distributions maintain a significant height between $105^{\circ}$ and $95^{\circ}$. For the two higher degrees of hydroxylation a similar, albeit smaller, relative broadening is observed for ReaxFF1 and ReaxFF2 both at the low $\left(100^{\circ}-105^{\circ}\right)$ and high $\left(115^{\circ}-120^{\circ}\right)$ ends of the $\mathrm{O}-\mathrm{Si}-\mathrm{O}$ distributions. As a few strained $(\mathrm{SiO})_{3}$ rings are only found in the low hydroxylated NPs and none of the clusters exhibit terminating defects, the reason for the observed broader distributions for the ReaxFF force fields may be due to the rigidity of the $\mathrm{SiO}_{4}$ 


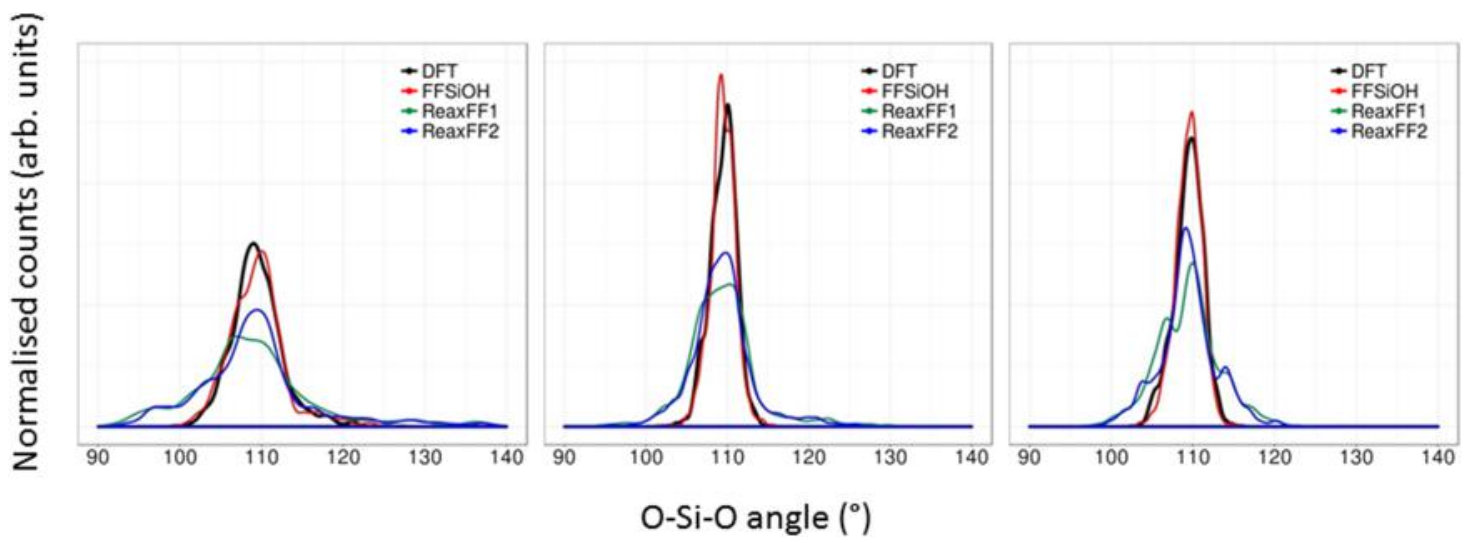

FIG. 3. Plots of the distribution of O-Si-O angles in $\left(\mathrm{SiO}_{2}\right)_{16}\left(\mathrm{H}_{2} \mathrm{O}\right)_{4} \mathrm{NPs}($ left $),\left(\mathrm{SiO}_{2}\right)_{16}\left(\mathrm{H}_{2} \mathrm{O}\right)_{6} \mathrm{NPs}$ (middle), and $\left(\mathrm{SiO}_{2}\right)_{16}\left(\mathrm{H}_{2} \mathrm{O}\right)_{8} \mathrm{NPs}(\mathrm{right})$ as optimised by DFT (black), FFSiOH (red), ReaxFF1 (green), and ReaxFF2 (blue).

sub-units being relatively lower than in the DFT or FFSiOH treatment.

\section{Si-O bond lengths}

The respective $\mathrm{Si}-\mathrm{O}$ distance distributions obtained for each method are found to hardly change with the degree of hydroxylation. Therefore in Fig. 4, we only report the results for the moderately hydroxylated (i.e., 37.5\%) NPs as a representative case. The $\mathrm{Si}-\mathrm{O}$ distances extracted from the DFT-optimised NPs have a very narrow distribution with values lying between 1.61 and $1.67 \AA$ with a mean value of $1.64 \AA$ and a weak shoulder for longer bond lengths. As for the case of the $\mathrm{O}-\mathrm{Si}-\mathrm{O}$ angle distribution, the $\mathrm{Si}-\mathrm{O}$ distance distribution from the FFSiOH-optimised NPs matches very well that from the DFT-optimised NPs, albeit exhibiting a very small relative shift in the weight of the distribution from the longer distances (1.65-1.67 $\AA$ ) to shorter distances (1.62-1.63 $\AA$ ). The distributions obtained from the NPs optimised using both ReaxFF force fields are quite distinct from the DFT-derived distribution, being broader, multi-peaked and having a shorter mean distance (1.58 $\AA$ for ReaxFF1 and $1.60 \AA$ for ReaxFF2). We note that the peak of the reference DFT-derived $\mathrm{Si}-\mathrm{O}$ distribution is slightly up-shifted with respect to a typical experimental $\mathrm{Si}-\mathrm{O}$ distribution for amorphous bulk silica, which sharply peaks at around $1.6 \AA^{57}$ This shift is likely partially due to the relatively small size of the considered NPs which possess a large proportion of surface atoms involved in relatively strained and thus slightly longer $\mathrm{Si}-\mathrm{O}$ bonds. However, optimisations of the structures of different silica polymorphs using DFT with the B3LYP functional also tend to systematically overestimate $\mathrm{Si}-\mathrm{O}$ bond lengths by $0.2-0.3 \AA$, which is also thus inherently parameterised into the performance of FFSiOH. ${ }^{36}$ The ReaxFF parameterisations also employ experimental data in their parameterisation helping us to explain their resulting broader downshifted $\mathrm{Si}-\mathrm{O}$ distributions.

\section{Si-O-Si angle}

Unlike the $\mathrm{O}-\mathrm{Si}-\mathrm{O}$ angle within $\mathrm{SiO}_{4}$ sub-units, the $\mathrm{Si}-\mathrm{O}-\mathrm{Si}$ angle presents a wider range of values commensurate with the relatively high degree of flexibility of the shared oxygen links between $\mathrm{SiO}_{4}$ tetrahedra. As for the $\mathrm{Si}-\mathrm{O}$ bond distance distributions, we focus on a characteristic $\mathrm{Si}-\mathrm{O}-\mathrm{Si}$ angle distribution derived from the set of moderately hydroxylated NPs (see Fig. 5). The DFT-derived distribution ranges between $120^{\circ}$ and $180^{\circ}$ and is skewed toward the lower angles with a maximum at around $137^{\circ}$. The $\mathrm{FFSiOH}$ derived distribution follows well the DFT-derived distribution with the same breadth and maximum peak position, with a slightly more pronounced peak coming from a small shift in weight of the distribution from $145^{\circ}$ to $160^{\circ}$ to around $137^{\circ}$. Both ReaxFF-derived distributions range between $\sim 130^{\circ}$ and $180^{\circ}$ and, unlike the DFT-derived distribution, are biased toward higher angles, with a maximum peak at $152^{\circ}$.

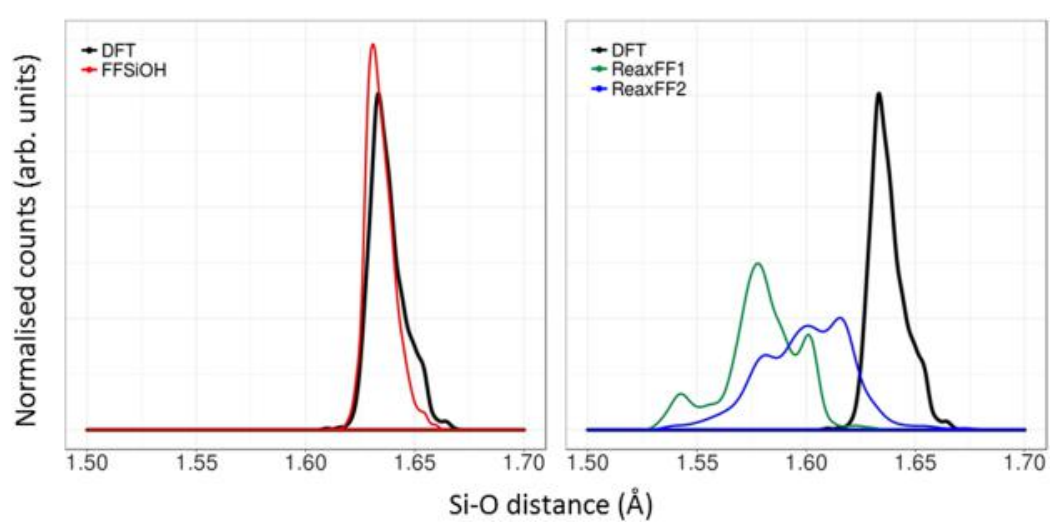

FIG. 4. Plots of the distribution of $\mathrm{Si}-\mathrm{O}$ bond lengths in $\left(\mathrm{SiO}_{2}\right)_{16}\left(\mathrm{H}_{2} \mathrm{O}\right)_{6} \mathrm{NPs}$ as optimised by DFT (black), FFSiOH (red-left), ReaxFF1 (green-right), and ReaxFF2 (blue-right). 


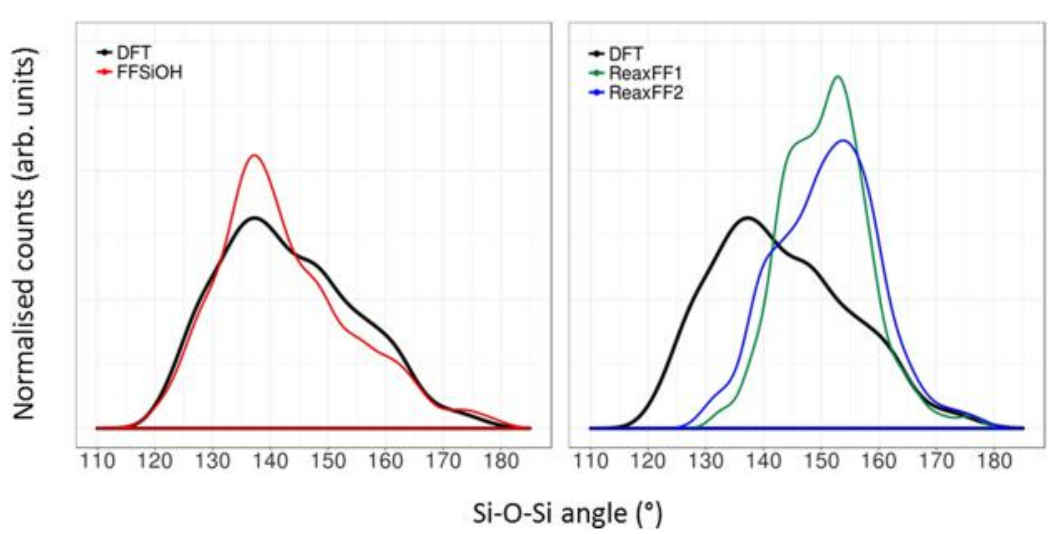

FIG. 5. Plots of the distribution of $\mathrm{Si}-\mathrm{O}-\mathrm{Si}$ angles in $\left(\mathrm{SiO}_{2}\right)_{16}\left(\mathrm{H}_{2} \mathrm{O}\right)_{6} \mathrm{NPs}$ as optimised by DFT (black), FFSiOH (red-left), ReaxFF1 (green-right), and ReaxFF2 (blue-right).
The ReaxFF-derived Si-O-Si angle distributions also appear to be the result of merging two fairly sharp sub-distributions which together mainly cover an angle range between $135^{\circ}$ and $170^{\circ}$. Conversely, the distribution coming from the DFToptimised NPs seems to be the result of a number of subdistributions which together mainly cover an angle range between $112^{\circ}$ and $170^{\circ}$.

\section{4. $\mathrm{OH} \ldots \mathrm{O}_{\text {siloxane }}$ distance}

The typical distribution of distances between hydrogen atoms of - $\mathrm{OH}$ groups and oxygen atoms in $\mathrm{Si}-\mathrm{O}-\mathrm{Si}$ bridges (i.e., $\mathrm{O}_{\text {siloxane }}$ ) does not change with the degree of hydroxylation. In Fig. 6, we show a typical distribution derived from our set of moderately hydroxylated NPs. In all distributions, two main peaks can be discerned. These peaks correspond to the distances between hydrogen atoms of a $\mathrm{Si}-\mathrm{OH}$ group and two distinct $\mathrm{O}_{\text {siloxane }}$ atoms bonded to the same $\mathrm{Si}$ atom. These two peaks appear at $3 \AA$ and $3.5 \AA$ in the DFT-derived distribution. The $\mathrm{OH} \cdots \mathrm{O}_{\text {siloxane }}$ distance distributions of NPs optimised using FFSiOH and ReaxFF1 underestimate the distances by $0.2 \AA$ and $0.4 \AA$, respectively. The FFSiOH distribution also has relatively higher more pronounced peaks than in the DFT-derived distribution. The distribution coming from NPs optimised using ReaxFF2 is the closest to that from the DFT-optimised NPs in terms of peak positions and peak heights, although the smaller distance peak appears to be the result of the merging of two smaller peaks with maxima slightly above and below $3.0 \AA ̊$.

\section{5. $\mathrm{OH} \ldots \mathrm{OH}$ distance}

The hydroxyl-hydroxyl $(\mathrm{OH} \cdots \mathrm{OH})$ distance is taken to be between a hydrogen atom of one $-\mathrm{OH}$ group and an oxygen atom of a neighbouring - $\mathrm{OH}$ group. The $\mathrm{OH} \cdots \mathrm{OH}$ distance distribution is found to change quite significantly with the degree of hydroxylation. In Fig. 7, we show the $\mathrm{OH} \cdots \mathrm{OH}$ distance distributions derived from clusters in our dataset with medium and high degrees of hydroxylation. At $37.5 \%$ hydroxylation, the DFT-derived distribution shows a broad double peaked structure with one maximum at $2.4 \AA$ and a slightly higher one at $3.7 \AA$. The distribution from the FFSiOH-optimised NPs recovers this type of distribution with a slight downward shift in the position of the $2.4 \AA$ peak to $2.3 \AA$. Both ReaxFF parameterisations also have peaks at distances corresponding to the two peaks of the DFTderived distribution. Unlike the DFT-derived distributions, however, these distributions also yield other peaks and have their weight strongly shifted to longer distances. For 50\% hydroxylation, the $\mathrm{OH} \cdots \mathrm{OH}$ distance distribution from the DFT-optimised NPs appears to consist of three broad peaks of increasing height centred at approximately $1.8 \AA 2.9 \AA$, and $3.5 \AA$. Distributions of this general type are reproduced from the NPs optimised using all three force fields. The distributions from the FFSiOH and ReaxFF2 data are particularly good in terms of the peak positions with respect to the DFTderived distribution. The ReaxFF1-derived distribution, however, exhibits more significantly downshifted peak positions with respect to the $2.9 \AA$ and $3.5 \AA$ peaks in the DFT-derived distribution.

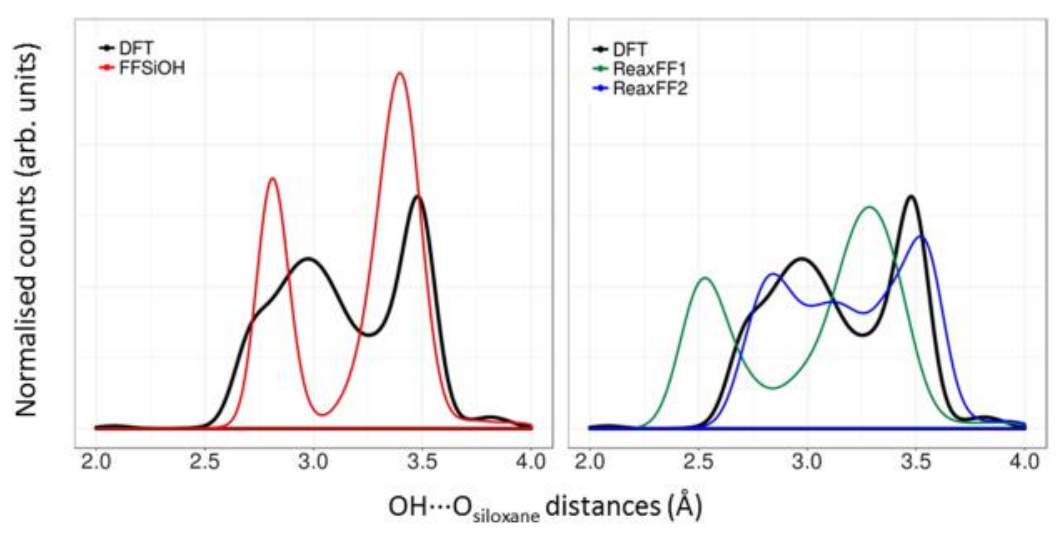

FIG. 6. Plots of the distribution of $\mathrm{OH} \cdots \mathrm{O}_{\text {siloxane }}$ distances in $\left(\mathrm{SiO}_{2}\right)_{16}\left(\mathrm{H}_{2} \mathrm{O}\right)_{6}$ NPs as optimised by DFT (black), FFSiOH (red-left), ReaxFF1 (green-right), and ReaxFF2 (blue-right). 


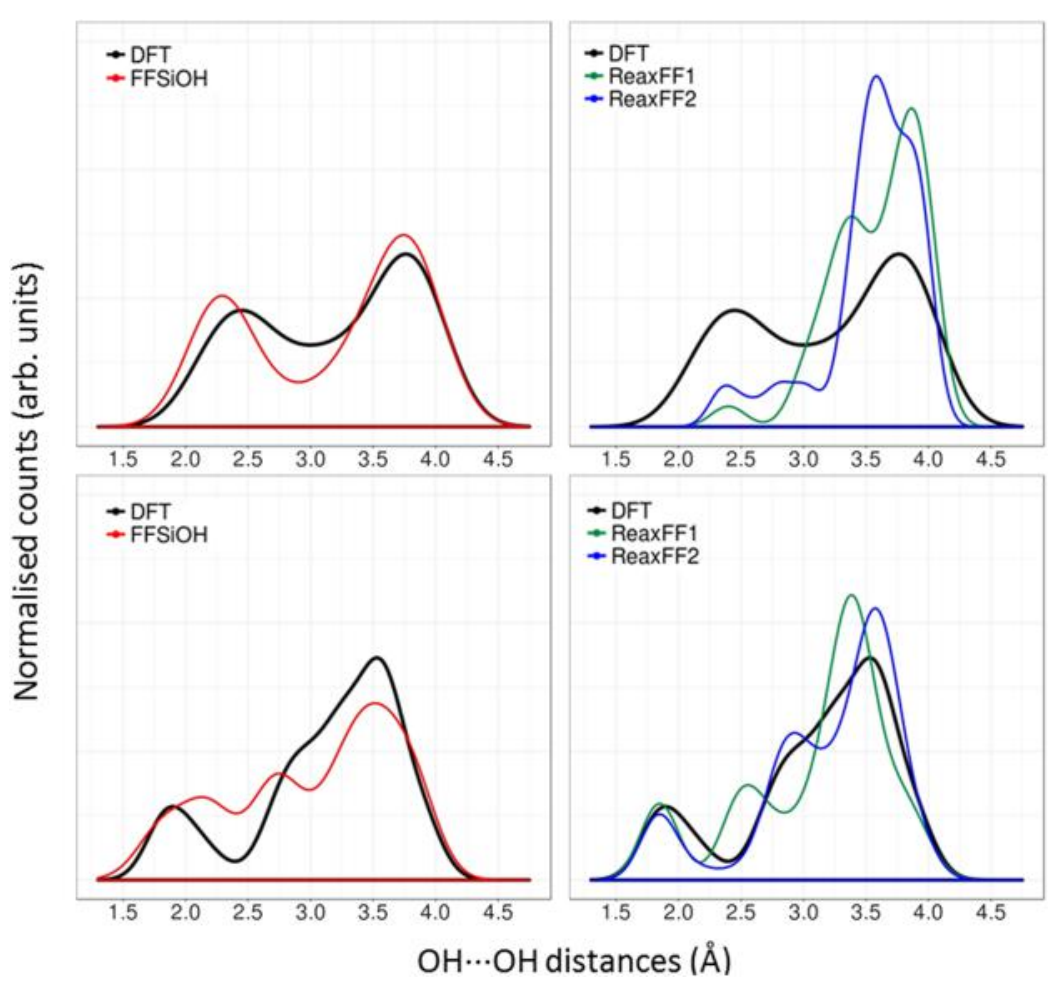

FIG. 7. Plots of the distribution of $\mathrm{OH} \cdots \mathrm{OH}$ distances in $\left(\mathrm{SiO}_{2}\right)_{16}\left(\mathrm{H}_{2} \mathrm{O}\right)_{6} \mathrm{NPs}$ (upper) and $\left(\mathrm{SiO}_{2}\right)_{16}\left(\mathrm{H}_{2} \mathrm{O}\right)_{8} \mathrm{NPs}$ (lower) NPs as optimised by DFT (black), FFSiOH (redleft), ReaxFF1 (green-right), and ReaxFF2 (blueright).

\section{Relative total energies}

In Fig. 8, we show the plots of relative total NP energies (i.e., the difference in the total energy of a nanocluster relative to the lowest energy nanocluster of the same composition) calculated using DFT against the corresponding energies calculated using the three considered force fields for low (a), moderate (b), and high (c) degrees of hydroxylation. In each case, a linear fit to the data is shown with its corresponding coefficient of determination $\left(\mathrm{r}^{2}\right)$. The data pertaining to all linear fits plotted in Fig. 8 are reported in Table I.

For the lowest degree of hydroxylation considered $(\mathrm{R}=0.25)$, the relative total energies of NPs optimised using FFSiOH mirror those from the DFT optimisations very well. Not only is there a good linear correlation between the FFSiOH and DFT data $\left(r^{2}=0.875\right)$ but the gradient of the linear fit is also very close to one with an intercept close to zero, indicating an excellent quantitative agreement between DFT and FFSiOH energies. For the same low degree of hydroxylation, total NP energies derived from both versions of ReaxFF show almost no correlation with the DFT derived data. The difference in the predictive power of FFSiOH and ReaxFF is nowhere else found to be so great. One can only speculate that the parameterisations of ReaxFF are biased toward silica with a moderate to high degree hydroxylation at the expense of reproducing the energetics of less hydroxylated systems.

For the set of moderately hydroxylated NPs, we indeed see a dramatic improvement for both versions of ReaxFF. For ReaxFF1, in particular, the linear correlation between the derived relative total energies and those derived from DFT is found to have a high coefficient of determination $\left(r^{2}=0.891\right)$. ReaxFF2 also shows a massive improvement $\left(r^{2}=0.773\right)$ with respect to its performance on the set of NPs with a low degree of hydroxylation. In both these cases, although the intercept is small, the gradient of the linear fit is similar and quite low $(<0.5)$ showing that the ReaxFF-derived energetic data are not in particularly good quantitative agreement with that from DFT. For FFSiOH, the performance is somewhat reduced with respect to that for the set of more weakly hydroxylated NPs, with a lower but reasonable linear correlation with the DFTderived data being found $\left(r^{2}=0.666\right)$. However, the gradient of the linear fit of the DFT versus FFSiOH data plot is fairly close to one (0.85) with an intercept close to zero, showing the capacity of FFSiOH for a reasonable quantitative reproduction of DFT-derived data.

For the highly hydroxylated NPs, none of the three force fields perform extremely well, with the total energies from ReaxFF2 having the best linear correlation with the DFT data $\left(r^{2}=0.596\right)$, slightly worse than for the moderately hydroxylated case. Although the gradient of the linear fit is fairly low, as for the fit for the moderate hydroxylation case, the intercept of the fit is quite close to zero. The performance of ReaxFF1, however, is significantly reduced with respect to that for the moderately hydroxylated NPs $\left(r^{2}=0.405\right)$ with an intercept close to $0.1 \mathrm{eV}$. Unlike its good/reasonable performance for the weakly and moderately hydroxylated NPs, respectively, FFSiOH performs worst of all for the highly hydroxylated NPs. Not only is the linear correlation with the DFT data not $\operatorname{good}\left(r^{2}=0.323\right)$, the linear fit has a relatively low gradient $(<0.35)$ with a significant non-zero intercept $(\sim 0.2 \mathrm{eV})$. This indicates that $\mathrm{FFSiOH}$ is not capturing something fundamental for highly hydroxylated NPs.

The significantly reduced performance of $\mathrm{FFSiOH}$ for highly hydroxylated NPs is consistent with some deficiency of the description of the $-\mathrm{OH}$ groups and their hydrogen bond interactions. Below, considering this possibility, we show how small changes could improve FFSiOH for these 
A) Low hydroxylation: $\left(\mathrm{SiO}_{2}\right)_{16}\left(\mathrm{H}_{2} \mathrm{O}\right)_{4}$

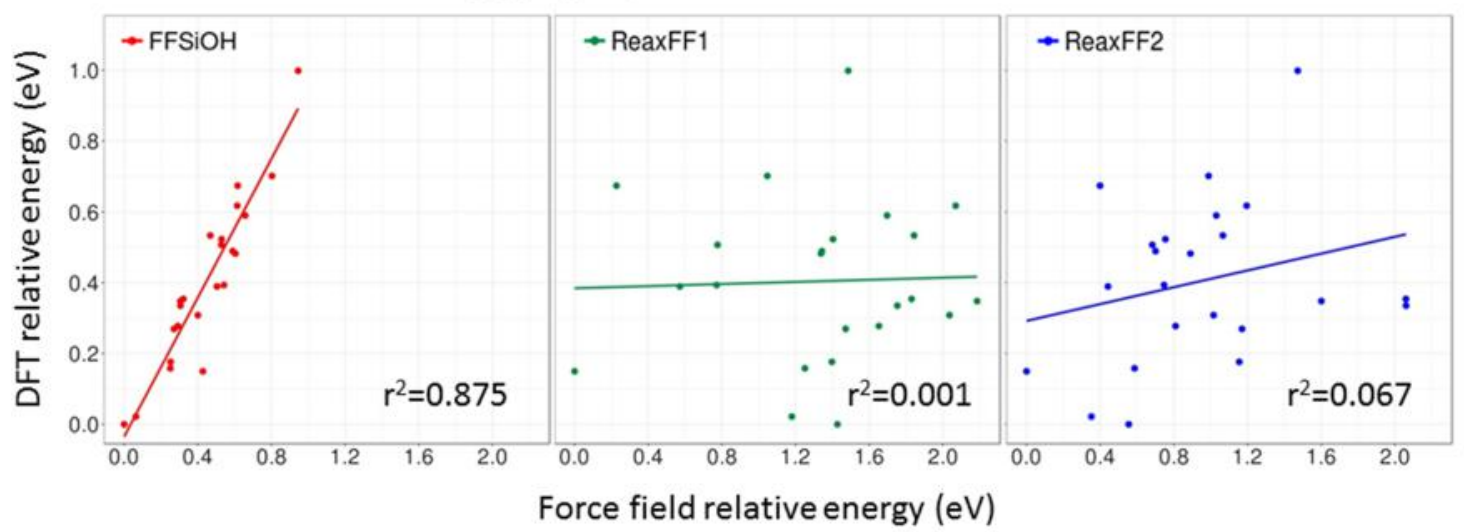

B) Moderate hydroxylation: $\left(\mathrm{SiO}_{2}\right)_{16}\left(\mathrm{H}_{2} \mathrm{O}\right)_{6}$

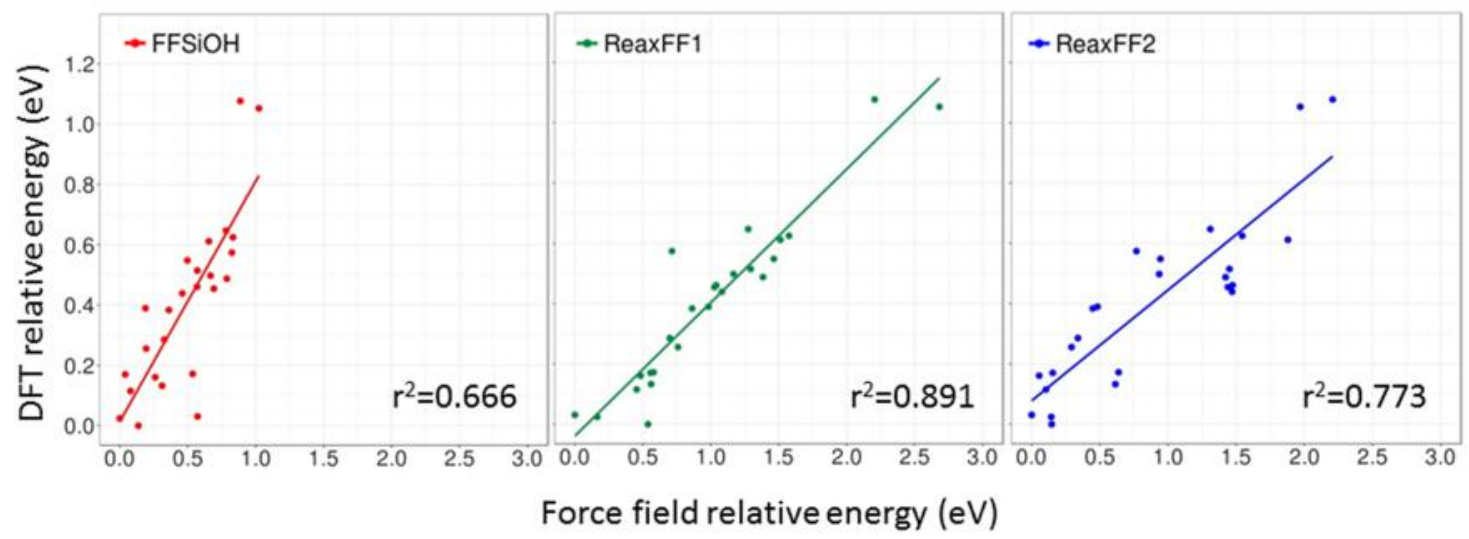

C) High hydroxylation: $\left(\mathrm{SiO}_{2}\right)_{16}\left(\mathrm{H}_{2} \mathrm{O}\right)_{8}$

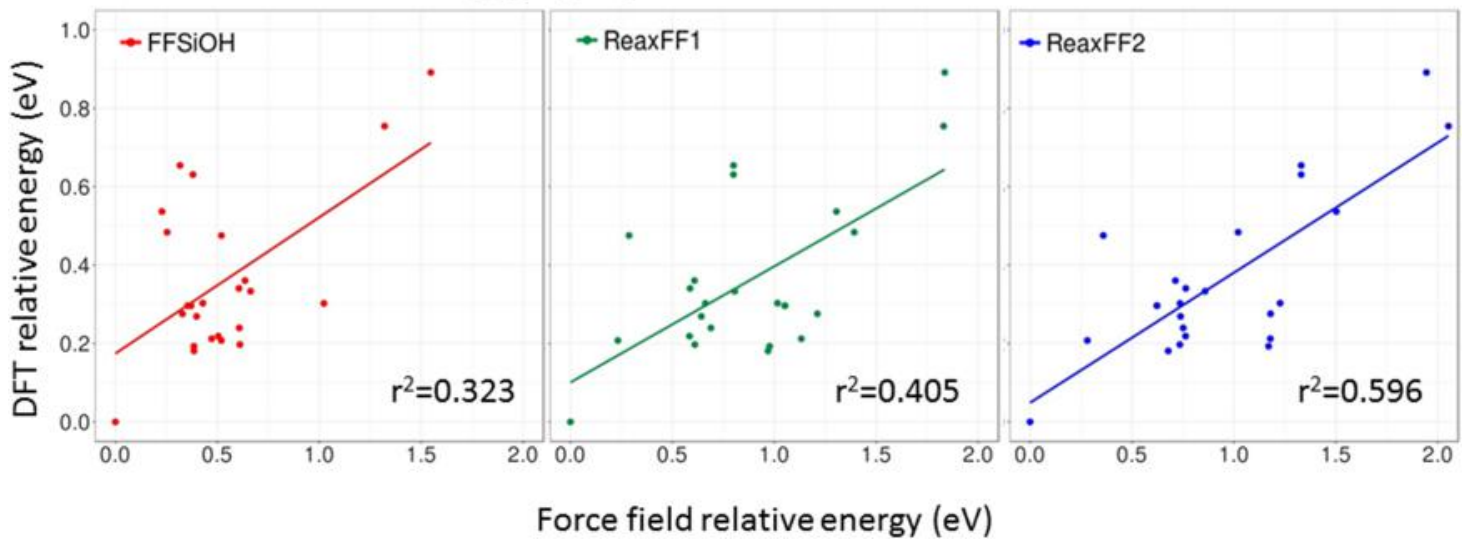

FIG. 8. Plots comparing the relative total energies of (a) $\left(\mathrm{SiO}_{2}\right)_{16}\left(\mathrm{H}_{2} \mathrm{O}\right)_{4} \mathrm{NPs}$, (b) $\left(\mathrm{SiO}_{2}\right)_{16}\left(\mathrm{H}_{2} \mathrm{O}\right)_{6} \mathrm{NPs}$, and (c) $\left(\mathrm{SiO}_{2}\right)_{16}\left(\mathrm{H}_{2} \mathrm{O}\right)_{8} \mathrm{NPs}$ as calculated by FFSiOH, ReaxFF, and ReaxFF2 with respect to the corresponding DFT-calculated data. The energy of the most energetically stable NP isomer as calculated by DFT is taken to be zero in each case.

cases. The poor performance of both parameterisations of ReaxFF for low hydroxylation is more difficult to evaluate. For low degrees of hydroxylation, NPs commonly display $(\mathrm{SiO})_{3}$ three-membered rings that have fairly high internal strain energies. ${ }^{47}$ If these rings are not accurately described, this could potentially disrupt the total energies of the NPs specifically for low degrees of hydroxylation. Thus far, however, our analysis could not find any link between the presence of three-membered rings and the ability of ReaxFF to reproduce DFT-derived total energies.

\section{Vibrational spectra}

In Fig. 9, we show the vibrational frequencies of three representative low energy NPs with low, moderate, and high degrees of hydroxylation, respectively, as calculated using the three considered force fields relative to the DFT-calculated data. The upper plots in Fig. 9 utilise logarithmic plots which tend to dampen discrepancies between the force field and DFT data when they are within a few percent of the absolute value of the vibrational frequency in question. Conversely, 
TABLE I. Values of $\mathrm{r}^{2}$, slope, and intercept for all plots of DFT relative energy versus force field relative energy in Fig. 8.

\begin{tabular}{|c|c|c|c|c|c|c|c|c|c|}
\hline \multirow{2}{*}{$\begin{array}{l}\text { Degree of } \\
\text { hydroxylation }(\%)\end{array}$} & \multicolumn{3}{|c|}{$\mathrm{FFSiOH}$} & \multicolumn{3}{|c|}{ ReaxFF1 } & \multicolumn{3}{|c|}{ ReaxFF2 } \\
\hline & $r^{2}$ & Slope & Intercept & $r^{2}$ & Slope & Intercept & $r^{2}$ & Slope & Intercept \\
\hline 25 (low) & 0.875 & 0.981 & -0.03 & 0.001 & 0.015 & 0.384 & 0.067 & 0.119 & 0.292 \\
\hline 37.5 (moderate) & 0.666 & 0.797 & 0.014 & 0.891 & 0.443 & -0.039 & 0.773 & 0.367 & 0.078 \\
\hline 50 (high) & 0.323 & 0.347 & 0.174 & 0.405 & 0.296 & 0.101 & 0.596 & 0.332 & 0.049 \\
\hline
\end{tabular}

such plots help us to quickly identify significant differences between the force field and DFT data. Of the three force fields, the vibrational frequencies calculated using FFSiOH are generally found to be closest to those calculated using DFT for all three degrees of hydroxylation and over all frequencies. Only for a small range of relatively low frequencies $\left(50-100 \mathrm{~cm}^{-1}\right)$ for the moderately hydroxylated NP are the ReaxFF1-calculated frequencies marginally better than the FFSiOH data relative to the DFT data. For the frequency range between 20 and $1000 \mathrm{~cm}^{-1}$, the ReaxFF-calculated vibrational frequencies reproduce reasonably well the DFTcalculated data, albeit with some underestimation of the DFT frequencies in the highly hydroxylated case. The ReaxFF2calculated frequencies also show an even greater degree of frequency underestimation for this system for this frequency range. For the $\left(\mathrm{SiO}_{2}\right)_{16}\left(\mathrm{H}_{2} \mathrm{O}\right)_{4} \mathrm{NP}$, ReaxFF2 also shows significant underestimation of the DFT-calculated frequencies in the range of $500-1000 \mathrm{~cm}^{-1}$. However, by far the main difference between the force field and DFT vibrational frequency data is for frequencies above $1000 \mathrm{~cm}^{-1}$. In the frequency range between 1000 and $1250 \mathrm{~cm}^{-1}$ (corresponding to the $\mathrm{Si}$ O stretch region), both ReaxFF parameterisations seriously overestimate the DFT-calculated frequencies by up to 400 $\mathrm{cm}^{-1}$. Additionally, for the hydroxyl O-H stretching frequencies in the range of $3750-3900 \mathrm{~cm}^{-1}$, the ReaxFF1-calculated frequencies typically diverge from the DFT frequencies by between 400 and $1000 \mathrm{~cm}^{-1}$, while the ReaxFF2-calculated frequencies tend to systematically underestimate the DFT frequencies by $400-800 \mathrm{~cm}^{-1}$. In comparison, the $\mathrm{O}-\mathrm{H}$ stretching frequencies calculated using FFSiOH are always found to be within $60 \mathrm{~cm}^{-1}$ of the DFT-calculated data.

More quantitatively, in Table II we show the calculated root mean square deviation (RMSD) of the vibrational data shown in Fig. 9 with respect to the DFT-calculated data for each

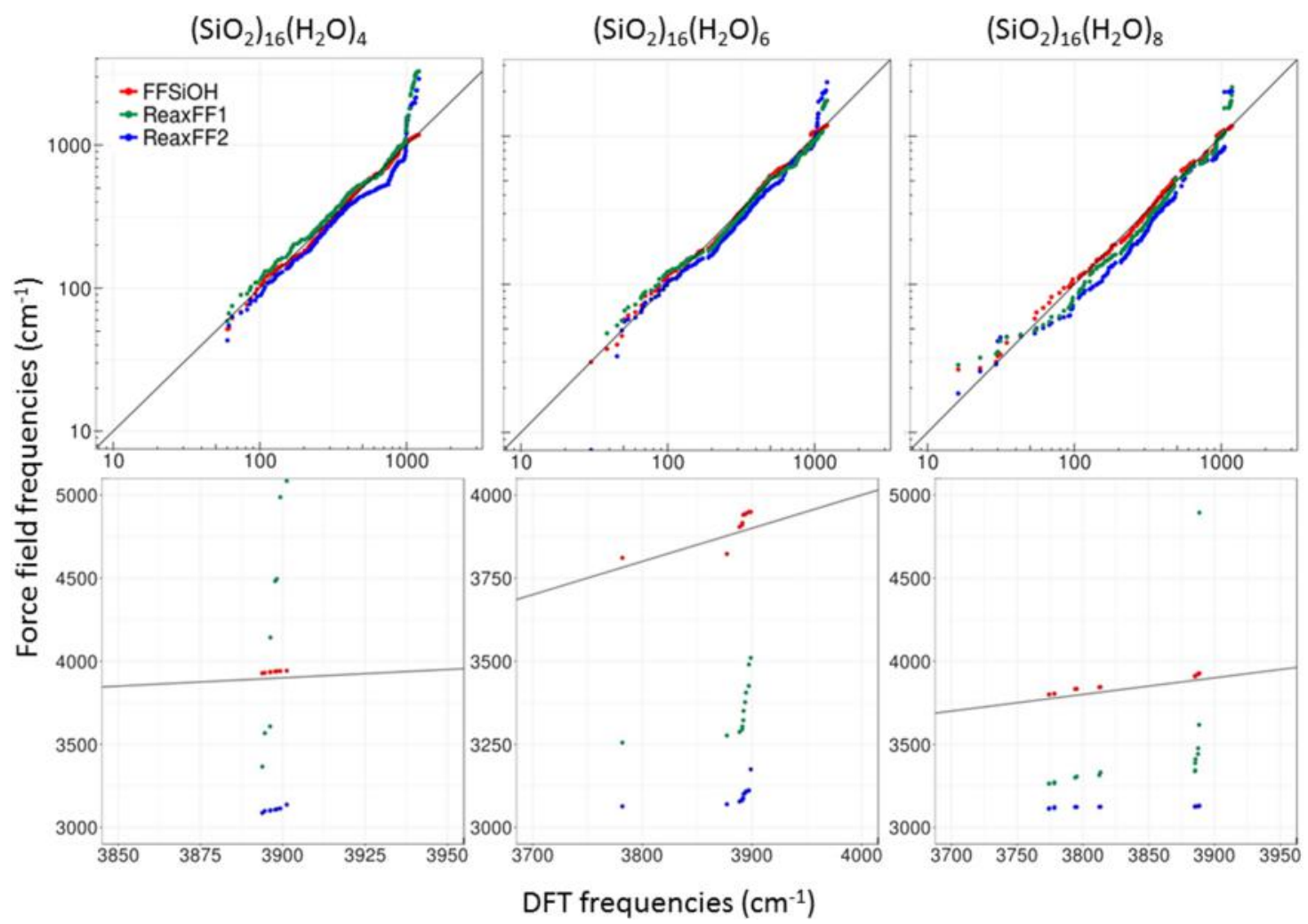

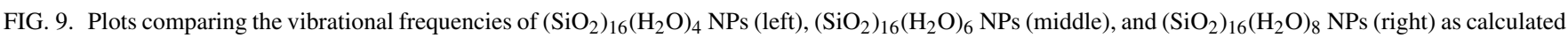
by FFSiOH (red), ReaxFF1 (green), and ReaxFF2 (blue) with respect to the corresponding DFT-calculated data. The upper three logarithmic plots show frequencies mainly associated with modes involving $\{\mathrm{Si}, \mathrm{O}\}$, whereas the lower plots focus on the high frequency range associated with $\mathrm{O}-\mathrm{H}$ stretching modes. The solid gray lines indicate where the data points would lie for perfect agreement between DFT and force field frequency values. 
TABLE II. Root mean square deviation (RMSD, $\mathrm{cm}^{-1}$ ) of the vibrational data calculated with each considered force field relative to the DFT-calculated data.

\begin{tabular}{lccc}
\hline \hline & FFSiOH & ReaxFF1 & ReaxFF2 \\
\hline$\left(\mathrm{SiO}_{2}\right)_{16}\left(\mathrm{H}_{2} \mathrm{O}\right)_{4}$ & 28.8 & 477.5 & 358.6 \\
$\left(\mathrm{SiO}_{2}\right)_{16}\left(\mathrm{H}_{2} \mathrm{O}\right)_{6}$ & 35.2 & 179.1 & 312.0 \\
$\left(\mathrm{SiO}_{2}\right)_{16}\left(\mathrm{H}_{2} \mathrm{O}\right)_{8}$ & 33.5 & 213.5 & 317.8 \\
\hline \hline
\end{tabular}

considered force field. Here the consistent good performance of FFSiOH is clearly highlighted by impressively low RMSD of the frequencies with respect to those calculated via DFT (in the range 28-36 for all three considered NPs). The frequencies from ReaxFF1 and ReaxFF2 exhibit relatively high RMSD values, with the highest values being 477.5 and $358.6 \mathrm{~cm}^{-1}$, respectively, for the NP with the lowest degree of hydroxylation Although ReaxFF2 maintains a similar level of performance for each degree of hydroxylation, ReaxFF1 shows some improvement in its performance for higher degrees of hydroxylation. Even in the best case for the moderately hydroxylated NP, the RMSD value of the ReaxFF1 vibrational data with respect to the DFT data is five times higher than that for the corresponding FFSiOH-calculated data.

\section{E. Proposed improvements for FFSiOH}

It is clear that a number of differences can be clearly identified between the structures, energies, and frequencies derived from the force field-calculated data and those derived from the DFT-calculated data. Although some of these differences may not be overly detrimental as our reference set is not perfect (e.g., in the case of $\mathrm{Si}-\mathrm{O}$ distance distributions), B3LYP-based DFT calculations are known to be particularly reliable for the relative energetics and vibrational frequencies of silica systems. Ideally, based on our analysis, one would thus like to be able to recommend ways in which to systematically improve the force fields for the treatment of hydroxylated silica NPs for these properties in particular. For either parameterisation of ReaxFF, this task is rather complex due to both: (i) the large number of terms in the force field that one should potentially consider and (ii) the diversity in the range of factors where significant differences between the DFT-derived properties and ReaxFFderived properties occur (e.g., relative NP isomer energies for low degrees of hydroxylation and $\mathrm{O}-\mathrm{H}$ vibrational frequencies). On the other hand, FFSiOH is based on fewer terms and, arguably, the main discrepancy with the DFT-derived properties is for the relative isomer energies for highly hydroxylated NPs.

The decline in the performance of FFSiOH for NPs with a high degree of hydroxylation has thus led us to test possible changes in the force field in order to improve the agreement with the DFT-derived data. Considering the fact that the disagreement with the DFT data is most apparent for highly hydroxylated NPs, we attempted the three following changes: (i) an increase of the cutoff distance for the hydrogen bond between hydroxyl-hydroxyl and between hydroxylsiloxane, (ii) lowering the hydroxyl-hydroxyl hydrogen bond interaction, and (iii) increasing the energy of the hydrogen bond with siloxane to double its original value. The results of each individual change, as well as the results of applying all changes at once, are summarized in Table III with regard to the linear fit of the newly derived relative energy data from the correspondingly modified-FFSiOH and DFT. Individually, lowering the hydroxyl-hydroxyl hydrogen bond interaction strength is found to be the change which gives the best improvement for the highly hydroxylated NPs. Here the value of $\mathrm{r}^{2}$ for the highest degree of hydroxylation increases up

TABLE III. Modifications to terms in the FFSiOH force field (column 1) and their impact for different degrees of hydroxylation (column 2), as measured by parameters describing the linear fit of resulting relative isomer energies with respect to the corresponding relative isomer energies derived from the DFT calculations (see also Fig. 8 for the results pertaining to the original FFSiOH parameterisation).

\begin{tabular}{lccrr}
\hline \hline FFSiOH modification & Degree of hydroxylation & $\mathrm{r}^{2}$ & Slope & $\mathrm{y}$-intercept \\
\hline Original & 25 (low) & 0.875 & 0.981 & -0.035 \\
& 37.5 (moderate) & 0.666 & 0.797 & 0.014 \\
& 50 (high) & 0.323 & 0.347 & 0.174 \\
\hline Increased H-bond cutoff & 25 (low) & 0.809 & 1.021 & 0.009 \\
& 37.5 (moderate) & 0.686 & 0.686 & 0.065 \\
& 50 (high) & 0.499 & 0.267 & 0.178 \\
\hline Lower -OH. . OH & 25 (low) & 0.883 & 0.990 & 0.009 \\
& 37.5 (moderate) & 0.678 & 0.868 & 0.065 \\
& 50 (high) & 0.703 & 0.446 & 0.178 \\
\hline Higher -OH siloxane & 25 (low) & 0.873 & 0.991 & -0.027 \\
& 37.5 (moderate) & 0.651 & 0.801 & 0.031 \\
& 50 (high) & 0.686 & 0.370 & 0.196 \\
\hline \hline All modifications & 25 (low) & 0.859 & 1.051 & 0.989 \\
& 37.5 (moderate) & 0.673 & 0.341 & -0.015 \\
& 50 (high) & 0.036 \\
& & 0.151 \\
\hline
\end{tabular}


to 0.703 from 0.323 for the original version of $\mathrm{FFSiOH}-$ see also Fig. 8(c). We note that this single change even slightly increases the $r^{2}$ values for NP relative energy data for low and medium hydroxylation. Although significant improvements can be made through other modifications (e.g., higher $\mathrm{OH}$-siloxane interaction) and their combinations, overall the simplest and most significant improvement is found simply through the change in $\mathrm{OH} \cdots \mathrm{OH}$ cutoff. We also note that an increased $\mathrm{OH}$-siloxane interaction, although formally improving FFSiOH in terms of relative energies, would be somewhat contrary to current chemical knowledge of the hydrophobicity of siloxanes in silica glass. This seems to indicate that the hydroxyl-hydroxyl interaction is the key factor to better improve FFSiOH so it can be applied to nanosilica systems with a greater range of hydroxylation. Further tests of the full implications of this modified version of $\mathrm{FFSiOH}$ are currently underway.

\section{CONCLUSIONS}

We have analysed FFSiOH and two versions of ReaxFF with respect to the hydroxylation energy, structure, relative energy of selected isomers, and vibrational spectra. We stress that our results should be interpreted in terms of how well the considered force fields perform with regard to only these properties, and as calculated using DFT. In particular, we do not compare with experimental data and we do not assess the full range of capabilities of ReaxFF (e.g., variable stoichiometries and reaction barriers). We also note that our investigation concerns small hydroxylated NPs and a hydroxylated nanoslab and thus should be extrapolated with due care to other types or sizes of system. However, these caveats noted, we feel that the chosen DFT data constitute a solid reference set and that our study thus represents a valid assessment of the performance of the considered force fields for treating hydroxylated nano-silica systems. Indeed, the very scarcity of the relevant experimental data for this size regime is what largely inspired the present comparative study.

In terms of hydroxylation energy, the general trends exhibited in the DFT-calculated data are well respected by the three force fields. ReaxFF1 parameters show an over-stabilization of structures with respect to size and hydroxylation, which the ReaxFF2 re-parameterisation seems to solve. As for structure, based on the geometrical features analysed, all force fields provide at least a reasonable description relative to that of the DFT calculations. FFSiOH, however, shows a particularly strong general agreement with the DFT-optimised NP structures. This implies that FFSiOH could be used as extremely efficient and reliable structure pre-optimiser upon which subsequent single point DFT calculations could be used to evaluate other properties (e.g., charge distributions, energetics, and NMR features). Such a FFSiOH//DFT(B3LYP) procedure has the huge advantage of performing the costly optimisation calculation at a classical level while the accuracy is ensured by DFT through a computationally cheap single energy evaluation.

In terms of relative energies, the degree of hydroxylation clearly shows limits on which force fields are reasonable to use under different circumstances. At low degree of hydroxylation,
FFSiOH gives the best results, but its performance begins to suffer as the degree of hydroxylation increases. On the other hand, both ReaxFF force fields fail badly at a lower degree of hydroxylation but give a good description for the medium hydroxylated regime, with the original ReaxFF1 parameters giving best results for this regime. At the highest degree of hydroxylation ( $\mathrm{R}=0.5)$, none of the force fields show a particularly good correlation with DFT data (ReaxFF2 performing the best of the three with $\mathrm{r}^{2}=0.596$ ).

For vibrational spectra, FFSiOH-derived values are found to be in very good agreement with the DFT-derived data, whereas ReaxFF-derived values show multiple deficiencies especially for high frequencies. One implication of the good performance of $\mathrm{FFSiOH}$ for calculating vibrational frequencies is that it could be used to compute the thermodynamic corrections needed for evaluating the free energy at almost negligible computational cost. In other words, one could compute heat capacities, entropies, and Gibbs free energies by correcting DFT-calculated internal electronic energies using the FFSiOH-calculated vibrational contributions.

Finally, we show how some small modifications to the FFSiOH parameterisation can improve its performance with respect to relative energies of highly hydroxylated NPs.

\section{SUPPLEMENTARY MATERIAL}

See supplementary material for structures and total energies of all hydroxylated nanosystems used. The structures are divided into the following two sets:

(A) Progressively hydroxylated nanosilica systems: $\left(\mathrm{SiO}_{2}\right)_{4}\left(\mathrm{H}_{2} \mathrm{O}\right)_{\mathrm{M}}, \quad\left(\mathrm{SiO}_{2}\right)_{8}\left(\mathrm{H}_{2} \mathrm{O}\right)_{\mathrm{M}}, \quad\left(\mathrm{SiO}_{2}\right)_{16}\left(\mathrm{H}_{2} \mathrm{O}\right)_{\mathrm{M}}$, and $\left(\mathrm{SiO}_{2}\right)_{24}\left(\mathrm{H}_{2} \mathrm{O}\right)_{\mathrm{M}}$ (see Refs. 41 and 42) and a hydroxylated nanoslab (see Ref. 45).

(B) The Hydroxylated Nanosilica Dataset (HND)composed of three sets of clusters specifically generated for this study with the respective stoichiometries:

(1) low hydroxylation- $\left(\mathrm{SiO}_{2}\right)_{16}\left(\mathrm{H}_{2} \mathrm{O}\right)_{4}$,

(2) moderate hydroxylation- $\left(\mathrm{SiO}_{2}\right)_{16}\left(\mathrm{H}_{2} \mathrm{O}\right)_{6}$,

(3) high hydroxylation- $\left(\mathrm{SiO}_{2}\right)_{16}\left(\mathrm{H}_{2} \mathrm{O}\right)_{8}$.

\section{ACKNOWLEDGMENTS}

Support from Spanish MINECO/FEDER Grant No. CTQ2015-64618-R grant and, in part, by Generalitat de Catalunya Grant No. 2014SGR97, XRQTC is acknowledged. We also acknowledge the NOMAD Center of Excellence project (this project has received funding from the European Union's Horizon 2020 research and innovation programme under Grant Agreement No. 676580). Access to supercomputer resources was provided through grants from the Red Española de Supercomputación.

\footnotetext{
${ }^{1}$ The Sol-Gel Handbook: Synthesis, Characterization and Applications, edited by D. Levy and M. Zayat (John Wiley \& Sons, 2015), Vol. 3, ISBN: 352767084X.

${ }^{2}$ C. J. Brinker and G. W. Scherer, Sol-Gel Science: The Physics and Chemistry of Sol-Gel Processing (Academic Press, 1990), ISBN: 9780121349707.

${ }^{3}$ H. Ehrlich, K. D. Demadis, O. S. Pokrovsky, and P. G. Koutsoukos, "Modern views on desilicification: Biosilica and abiotic silica dissolution in natural and artificial environments," Chem. Rev. 110, 4656-4689 (2010).
} 
${ }^{4}$ P. Aggarwal, R. Singh Pratap, and Y. Aggarwal, "Use of nano-silica in cement based materials-A review," Cogent Environ. 2(1-11), 1078018 (2015).

${ }^{5}$ S. Zhoua, L. Wu, J. Suna, and W. Shen, "The change of the properties of acrylic-based polyurethane via addition of nano-silica," Prog. Org. Coat. 45, 33-42 (2002).

${ }^{6}$ M. Qu, J. S. Meth, G. S. Blackman, G. M. Cohen, K. G. Sharp, and K. J. Van Vliet, "Tailoring and probing particle-polymer interactions in PMMA/silica nanocomposites," Soft Matter 7, 8401-8408 (2011).

${ }^{7}$ C. S. Cundya and P. A. Cox, "The hydrothermal synthesis of zeolites: Precursors, intermediates and reaction mechanism," Microporous Mesoporous Mater. 82, 1-78 (2005).

${ }^{8}$ R. Hellmann, R. Wirth, D. Daval, J.-P. Barnes, J.-M. Penisson, D. Tisserand, T. Epicier, B. Florin, and R. L. Hervig, "Unifying natural and laboratory chemical weathering with interfacial dissolution-reprecipitation: A study based on the nanometer-scale chemistry of fluid-silicate interfaces," Chem. Geol. 294-295, 203-216 (2012).

${ }^{9}$ R. Hellmann, S. Cotte, E. Cadel, S. Malladi, L. S. Karlsson, S. LozanoPerez, M. Cabié, and A. Seyeux, "Nanometre-scale evidence for interfacial dissolution-reprecipitation control of silicate glass corrosion," Nat. Mater. 14, 307-311 (2015).

${ }^{10}$ E. F. van Dishoeck, "Astrochemistry of dust, ice and gas: Introduction and overview," Faraday Discuss. 168, 9-47 (2014).

${ }^{11}$ R. K. Iler, The Chemistry of Silica (John Wiley and Sons, 1979).

${ }^{12}$ Y. Wang, M. Y. He, and R. Y. Chen, "Fabrication of mechanically robust antireflective films using silica nanoparticles with enhanced surface hydroxyl groups," J. Mater. Chem. A 3, 1609-1618 (2015).

${ }^{13}$ P. Chandra, D. S. Doke, S. B. Umbarkar, K. Vank, and A. V. Biradar, "Silica microspheres containing high density surface hydroxyl groups as efficient epoxidation catalysts," RSC Adv. 5, 21125-21131 (2015).

${ }^{14}$ B. Yang, S. Shaikutdinov, and H.-J. Freund, "Tuning Spatial Distribution of Surface Hydroxyls on a Metal-Supported Single-Layer Silica,” J. Phys. Chem. Lett. 5, 1701-1704 (2014).

${ }^{15}$ H. Zhang, D. R. Dunphy, X. Jiang, H. Meng, B. Sun, D. Tarn, M. Xue, X. Wang, S. Lin, Z. Ji, R. Li, F. L. Garcia, J. Yang, M. L. Kirk, T. Xia, J. I. Zink, A. Nel, and C. J. Brinker, "Processing pathway dependence of amorphous silica nanoparticle toxicity: Colloidal vs pyrolytic," J. Am. Chem. Soc. 134, 15790-15804 (2012).

${ }^{16}$ A. Marucco, F. Turci, L. O’Neill, H. J. Byrne, B. Fubini, and I. Fenoglio, "Hydroxyl density affects the interaction of fibrinogen with silica nanoparticles at physiological concentration," J. Colloid Interface Sci. 419, 86-94 (2014).

${ }^{17}$ A. Rimola, D. Costa, M. Sodupe, J.-F. Lambert, and P. Ugliengo, "Silica surface features and their role in the adsorption of biomolecules: Computational modeling and experiments," Chem. Rev. 113, 4216-4313 (2013).

${ }^{18}$ F. Musso, P. Mignon, P. Ugliengo, and M. Sodupe, "Cooperative effects at water-crystalline silica interfaces strengthen surface silanol hydrogen bonding. An ab initio molecular dynamics study," Phys. Chem. Chem. Phys. 14, 10507-10514 (2012).

${ }^{19}$ F. Musso, P. Ugliengo, and M. Sodupe, "Do H-bond features of silica surfaces affect the $\mathrm{H}_{2} \mathrm{O}$ and $\mathrm{NH}_{3}$ adsorption? Insights from periodic B3LYP calculations," J. Phys. Chem. A 115, 11221-11228 (2011).

${ }^{20}$ D. Makimura, C. Metin, T. Kabashima, T. Matsuoka, Q. P. Nguyen, and C. R. Miranda, "Combined modeling and experimental studies of hydroxylated silica nanoparticles," J. Mater. Sci. 45, 5084-5088 (2010).

${ }^{21}$ P. M. Dove, N. Han, A. F. Wallace, and J. J. de Yoreo, "Kinetics of amorphous silica dissolution and the paradox of the silica polymorphs," Proc. Natl. Acad. Sci. U. S. A. 105, 9903-9908 (2008).

${ }^{22}$ L. T. Zhuravlev, "The surface chemistry of amorphous silica. Zhuravlev model," Colloids Surf., A 173, 1-38 (2000).

${ }^{23}$ M. J. Mora-Fonz, C. R. A. Catlow, and D. W. Lewis, "Oligomerization, and cyclization processes in the nucleation of microporous silicas," Angew. Chem., Int. Ed. 44, 3082-3086 (2005).

${ }^{24}$ T. T. Trinh, A. P. J. Jansen, and R. A. van Santen, "Mechanism of oligomerization reactions of silica," J. Phys. Chem. B 110, 23099-23106 (2006).

${ }^{25}$ C. L. Schaffer and K. T. Thomson, "Density functional theory investigation into structure and reactivity of prenucleation silica species," J. Phys. Chem. C 112, 12653-12662 (2008).

${ }^{26}$ H.-P. Cheng, R. N. Bartlett, and U. Landman, "Structure, collective hydrogen transfer, and formation of $\mathrm{Si}(\mathrm{OH})_{4}$ in $\mathrm{SiO}_{2}-\left(\mathrm{H}_{2} \mathrm{O}\right)_{n}$ clusters," J. Chem. Phys. 116, 9300-9304 (2002).
${ }^{27}$ J. Yeon and A. C. T. van Duin, "ReaxFF molecular dynamics simulations of hydroxylation kinetics for amorphous and nano-silica structure, and its relations with atomic strain energy," J. Phys. Chem. C 120, 305-3017 (2016).

${ }^{28}$ S. Bhattacharya and J. Kieffer, "Molecular dynamics simulation study of growth regimes during polycondensation of silicic acid: From silica nanoparticles to porous gels," J. Phys. Chem. C 112, 1764-1771 (2008).

${ }^{29}$ J. C. Fogarty, H. M. Aktulga, A. Y. Grama, A. C. T. van Duin, and S. A. Pandit, "A reactive molecular dynamics simulation of the silica-water interface," J. Chem. Phys. 132, 174704 (2010).

${ }^{30}$ J. Du and A. N. Cormack, "Molecular dynamics simulation of the structure and hydroxylation of silica glass surfaces," J. Am. Ceram. Soc. 88, 2532-2539 (2005)

${ }^{31}$ A. A. Hassanali and S. J. Singer, "Model for the water-amorphous silica interface: The undissociated surface,” J. Phys. Chem. B 111, 11181-11193 (2007).

${ }^{32}$ P. E. M. Lopes, V. Murashov, M. Tazi, E. Demchuk, and A. D. MacKerell, "Development of an empirical force field for silica. Application to the quartz-water interface," J. Phys. Chem. B 110, 2782-2792 (2006).

${ }^{33}$ F. S. Emami, V. Puddu, R. J. Berry, V. Varshney, S. V. Patwardhan, C. C. Perry, and H. Heinz, "Force field and a surface model database for silica to simulate interfacial properties in atomic resolution," Chem. Mater. 26, 2647-2658 (2014).

${ }^{34}$ E. R. Cruz-Chu, A. Aksimentiev, and K. Schulten, "Water-silica force field for simulating nanodevices," J. Phys. Chem. B 110, 21497-21508 (2006).

${ }^{35}$ T. S. Mahadeven and S. H. Garofalini, "Dissociative chemisorption of water onto silica surfaces and formation of hydronium ions," J. Phys. Chem. C 112, 1507-1515 (2008).

${ }^{36}$ A. Pedone, G. Malavasi, M. C. Menziani, U. Segre, F. Musso, M. Corno, B. Civalleri, and P. Ugliengo, "FFSiOH: A new force field for silica polymorphs and their hydroxylated surfaces based on periodic B3LYP calculations," Chem. Mater. 20, 2522-2531 (2008).

${ }^{37}$ R. T. Cygan, J.-J. Liang, and A. G. Kalinichev, "Molecular models of hydroxide, oxyhydroxide, and clay phases and the development of a general force field," J. Phys. Chem. B 108, 1255-1266 (2004).

${ }^{38} \mathrm{Z}$. Du and N. H. de Leeuw, "A combined density functional theory and interatomic potential-based simulation study of the hydration of nano-particulate silicate surfaces," Surf. Sci. 554, 193-210 (2004).

${ }^{39}$ Y. He, C. Cao, Y.-X. Wan, and H.-P. Cheng, "From cluster to bulk: Size dependent energetics of silica and silica-water interaction," J. Chem. Phys. 124, 024722 (2006)

${ }^{40}$ S. T. Bromley and E. Flikkema, "Novel structures and energy spectra of hydroxylated $\left(\mathrm{SiO}_{2}\right)_{8}$-based clusters: Searching for the magic $\left(\mathrm{SiO}_{2}\right)_{8} \mathrm{O}_{2} \mathrm{H}_{3}$ cluster," J. Chem. Phys. 122, 114303 (2005).

${ }^{41}$ K. E. Jelfs, E. Flikkema, and S. T. Bromley, "Hydroxylation of silica nanoclusters $\left(\mathrm{SiO}_{2}\right)_{\mathrm{M}}\left(\mathrm{H}_{2} \mathrm{O}\right)_{\mathrm{N}}, \mathrm{M}=4,8,16,24$ : Stability and structural trends," Phys. Chem. Chem. Phys. 15, 20438-20443 (2013).

${ }^{42}$ E. Flikkema, K. E. Jelfs, and S. T. Bromley, "Structure and energetics of hydroxylated silica clusters, $\left(\mathrm{SiO}_{2}\right)_{\mathrm{M}}\left(\mathrm{H}_{2} \mathrm{O}\right)_{\mathrm{N}}, \mathrm{M}=8,16$ and $\mathrm{N}=1-4$ : $\mathrm{A}$ global optimisation study," Chem. Phys. Lett. 554, 117-122 (2012).

${ }^{43}$ K. E. Jelfs, E. Flikkema, and S. T. Bromley, "Evidence for atomic mixing via multiple intermediates during the dynamic interconversion of silicate oligomers in solution," Chem. Commun. 48, 46-48 (2012).

${ }^{44}$ P. J. Stephens, F. J. Devlin, C. F. Chabalowski, and M. J. Frisch, "Ab initio calculation of vibrational absorption and circular dichroism spectra using density functional force fields," J. Phys. Chem. 98, 11623-11627 (1994).

${ }^{45}$ P. Ugliengo, M. Sodupe, F. Musso, I. J. Bush, R. Orlando, and R. Dovesi, "Realistic models of hydroxylated amorphous silica surfaces and MCM-41 mesoporous material simulated by large-scale periodic B3LYP calculations," Adv. Mater. 20, 4579-4583 (2008).

${ }^{46}$ M. J. Frisch et al., GAUSSIAN 09, Revision D.01, Gaussian, Inc., Wallingford, 2013.

${ }^{47}$ S. T. Bromley, I. de P. R. Moreira, F. Illas, and J. C. Wojdeł, "Importance of the embedding environment on the strain within small rings in siliceous materials," Phys. Rev. B 73, 134202 (2006).

${ }^{48}$ J. D. Gale and A. L. Rohl, "The general utility lattice program (GULP)," Mol. Simul. 29, 291-341 (2003).

${ }^{49}$ E. Flikkema and S. T. Bromley, "Defective to fully coordinated crossover in complex directionally bonded nanocluster," Phys. Rev. B 80, 035402 (2009).

${ }^{50}$ E. Flikkema and S. T. Bromley, "Dedicated global optimization search for ground state silica nanoclusters: $\left(\mathrm{SiO}_{2}\right)_{\mathrm{N}}(\mathrm{N}=6-12)$," J. Phys. Chem. B 108, 9638 (2004). 
${ }^{51}$ M. A. Zwijnenburg, F. Illas, and S. T. Bromley, "Long range coupling between defect centres in inorganic nanostructures: Valence alternation pairs in nanoscale silica," J. Chem. Phys. 137, 154313 (2012).

${ }^{52}$ T. Walsh, M. Wilson, and A. P. Sutton, "Hydrolysis of the amorphous silica surface. II. Calculation of activation barriers and mechanisms," J. Chem. Phys. 113, 9191 (2000).

${ }^{53} \mathrm{P}$. Masini and M. Bernasconi, "Ab initio simulations of hydroxylation and dehydroxylation reactions at surfaces: Amorphous silica and brucite," J. Phys.: Condens. Matter 14, 4133 (2002).
${ }^{54}$ M.-H. Du, A. Kolchin, and H.-P. Cheng, "Water-silica surface interactions: A combined quantum-classical molecular dynamic study of energetics and reaction pathways," J. Chem. Phys. 119, 6418 (2003).

${ }^{55}$ Y. Ma, A. S. Foster, and R. M. Nieminen, "Reactions and clustering of water with silica surface," J. Chem. Phys. 122, 144709 (2005).

${ }^{56}$ A. Rimola and P. J. Ugliengo, "A quantum mechanical study of the reactivity of $(\mathrm{SiO})_{2}$-defective silica surfaces," J. Chem. Phys. 128, 204702 (2008). 